\title{
Large Scale Assembly and Characterization of Bi-2223 HTS Conductors
}

\author{
A. Ballarino, L. Martini, S. Mathot, T. Taylor, and R. Brambilla
}

\begin{abstract}
The powering of the LHC machine requires more than 1000 High Temperature Superconducting (HTS) current leads. These leads contain, at their cold end, HTS conductors made of stacks of Bi-2223 tape with gold-doped silver matrix. CERN specified and purchased $31 \mathrm{~km}$ of this material, which was delivered on spools in unit lengths of 100 to $300 \mathrm{~m}$. On reception the tape was inspected, cut into short length and vacuum soldered into stacks. All stacks were characterized in liquid nitrogen using a measuring procedure and set-up specifically developed for this purpose. Contact resistance values and critical currents at different electric field criteria were measured, from which the n-values have been extrapolated from the experimental V-I characteristics. This paper reports on the assembly and electrical characterization (up to $800 \mathrm{~A}$ ) of more than ten thousand $\mathrm{Bi}-2223$ stacks. Three types of stack were made from tape from two manufacturers. The assembly and soldering procedures and the set-up for the series electrical characterization are described. An analysis of the measured data is presented, with particular regard to the homogeneity of the electrical properties of both the tape and the derived stacks.
\end{abstract}

Index Terms-Bi-2223 conductors, critical current, current leads, high-temperature superconductors.

\section{INTRODUCTION}

$\mathbf{T}$ HE powering of the LHC accelerator is made via over 1000 High Temperature Superconducting (HTS) current leads. These leads incorporate HTS material in the form of stacks of Bi-2223 tapes with a gold-doped silver matrix. The design of the LHC leads is such that the HTS material operates between $50 \mathrm{~K}$ and the $4.5 \mathrm{~K}$ liquid helium bath [1].

Following an extensive R\&D program, CERN specified and purchased $31 \mathrm{~km}$ of Bi-2223 tape for the HTS lead project [2]. Two manufacturers supplied the tape, for which thermal, electrical and mechanical characteristics all met the specified requirements. The assembly of tapes into stacks was made at CERN, where the tape, delivered on spools, was visually inspected to identify and isolate potential local defects, cut into short length and vacuum soldered to form rugged conductor stacks to be supplied to the lead manufacturers. The stacks, of which over ten thousand have been produced, were all electrically characterized at liquid nitrogen temperature. This was

Manuscript received August 25, 2006.

A. Ballarino, S. Mathot, and T. Taylor are with the European Organization for Nuclear Research (CERN), 1211 Geneva 23, Switzerland (e-mail: amalia. ballarino@cern.ch; serge.mathot@cern.ch; tom.taylor@cern.ch).

L. Martini and R. Brambilla are with CESI Ricerca Spa, Milano, Italy (e-mail: lmartini@cesiricerca.it; rlbrambilla@cesiricerca.it).

Color versions of one or more of the figures in this paper are available online at http://ieeexplore.ieee.org.

Digital Object Identifier 10.1109/TASC.2007.900043
TABLE I

PROPERTIES OF Bi-2223 TAPE USED IN LHC LEADS

\begin{tabular}{lllll}
\hline \hline & & & \multicolumn{1}{c}{ AMSC } & EHTS \\
\hline Total tape length & $L$ & $\mathrm{~km}$ & 14 & 17 \\
Total number of spools & $N S$ & & 109 & 92 \\
Number of HTS filaments & $N f$ & & 55 & 121 \\
Matrix gold percentage & $A u \%$ & $\mathrm{wt}$ & 5.3 & 5 \\
Tape width & $W$ & $\mathrm{~mm}$ & $4 \pm 0.4$ & $4 \pm 0.4$ \\
Tape thickness & $T h$ & $\mathrm{~mm}$ & $0.215 \pm 0.02$ & $0.22 \pm 0.03$ \\
Filling factor & $f$ & $\%$ & 40 & 30 \\
Tape $n$-value at 77 K & $n$ & & 20 & 30 \\
Young's modulus & $E$ & $\mathrm{GPa}$ & 70 & 60 \\
Tensile strength & $S t r$ & $\mathrm{MPa}$ & 50 & 100 \\
Critical bending radius & $R$ & $\mathrm{~mm}$ & 100 & 50 \\
Critical current at 77 K & $I_{C}$ & $\mathrm{~A}$ & $>100$ & $\geq 70$ \\
\hline \hline
\end{tabular}

done at CESI according to a CERN specification. The results of the measurements on the stacks, individually identified by CERN, were stored in a purpose-built database.

Current leads are a commercially viable application of HTS material, and the CERN LHC application is large scale. The lead project has provided a unique opportunity to evaluate the results of the corresponding industrialization, and this paper covers the aspects of the manufacture of the stacks and the electrical performance and homogeneity of both the tape and the stacks. The study includes a comparison of results of measurements performed on the Bi-2223 stacks with calculations based on the performance of the constituent tape.

\section{MAterial AND Test Set-UP}

\section{A. HTS Tape}

The Bi-2223 multi-filamentary tape was supplied to CERN on spools in convenient lengths of 100 to $300 \mathrm{~m}$. The two suppliers, AMSC and EHTS, were required to perform critical current measurements on each spool of tape. Mechanical (Young's modulus, $E$, tensile strength at room temperature, Str, and bending radius giving a critical current degradation of less than $5 \%, R$ ) and filling factor $(f)$ measurements were made on four short samples extracted from each unit length, the unit length being defined as the length of tape obtained from the same billet and that underwent, during production, the same mechanical and thermal processes. The properties of the whole production of tape are summarized in Table I. The pitch increment for the critical current $\left(I_{c}\right)$ measurements was 0.5 $\mathrm{m}$ for the EHTS tape and $10 \mathrm{~m}$ for the AMSC tape, whereas for the geometrical measurements it was respectively $0.01 \mathrm{~m}$ and $0.07 \mathrm{~m}$. In Table I, $L$ is the total length delivered, $N s$ the total number of spools, $N f$ the number of superconducting filaments, Au\% the percentage weight of gold in the silver 

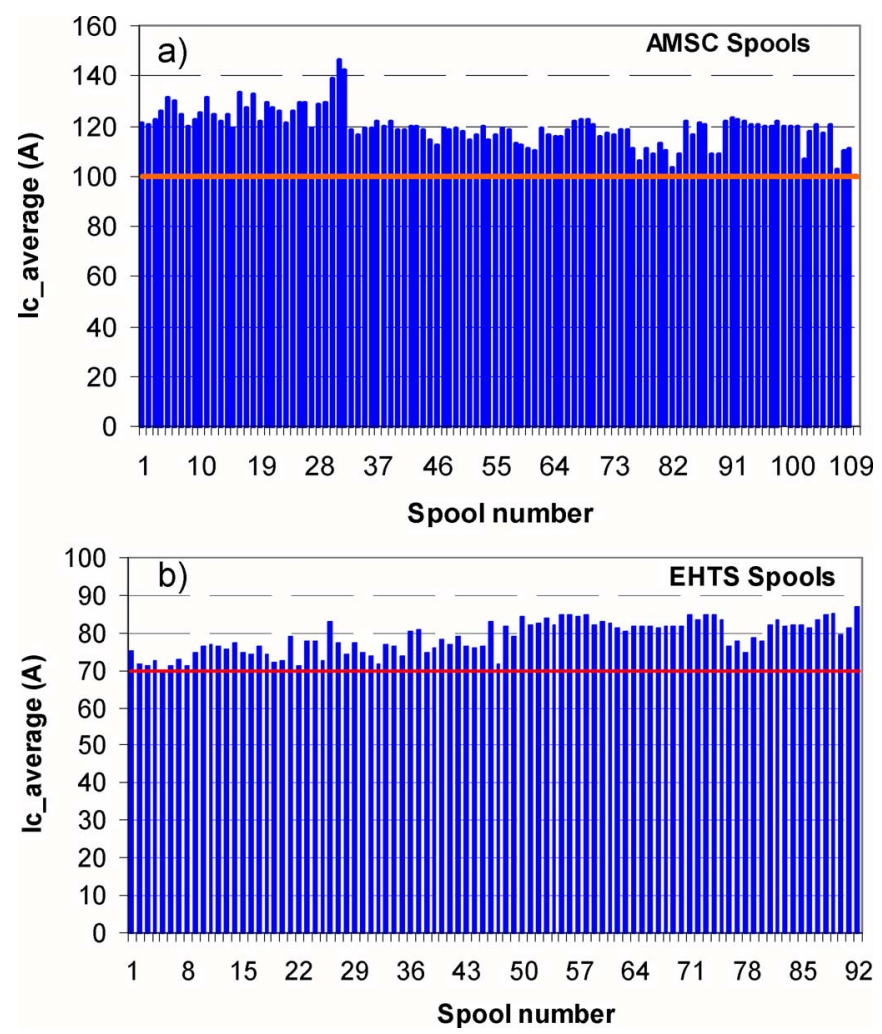

Fig. 1. Average critical current (Ic_average) measured on different spools of AMSC (a) and EHTS (b) Bi-2223 tape.

matrix, $W$ and $T h$ the tape width and thickness, and $n$ the average $n$-value of the $V-I$ characteristic at $77 \mathrm{~K}$ and in self-field. The average critical currents measured on each spool $(1 \mu \mathrm{V} / \mathrm{cm}$ criterion, $77 \mathrm{~K}$, self-field) are given in Fig. 1. The minimum, maximum, and average critical currents were 103 , 147, and 120 A for AMSC tape, and 70, 87, and 79 A for EHTS tape; coefficients of variation were 5.8 and $5 \%$ respectively.

\section{B. Stacking Procedure}

Stacks of $0.35 \mathrm{~m}$ in length were made by soldering together tapes using $96.5 \mathrm{Sn}-3.5 \mathrm{Ag}$ eutectic alloy. Vacuum soldering technology was adopted and no flux was used to avoid the delicate post-soldering cleaning operation and the potential long term corrosion due to flux residues. The tapes, cut to the required length and inspected, were cleaned with soft abrasive paper and high purity alcohol and assembled in graphite molds with interleaved solder foils. Soldering of 60 stacks per cycle was done in a horizontal all-metal vacuum furnace with a peak temperature of $240^{\circ} \mathrm{C}$ lasting for 15 minutes. In spite of the relatively long thermal cycle (about 1 hour), no evidence of excessive diffusion of solder was observed.

Three types of stack were made: (1) with 8 AMSC tapes (AMSC-8), (2) with 7 EHTS tapes (EHTS-7) and (3) with 9 EHTS tapes (EHTS-9). These stacks were assigned to leads operating at different current ratings.

During transport and storage, $\mathrm{Bi}-2223$ tapes and stacks were enclosed in specially designed protection boxes, packaged and sealed in nitrogen gas or vacuum to protect the surface against oxidation.
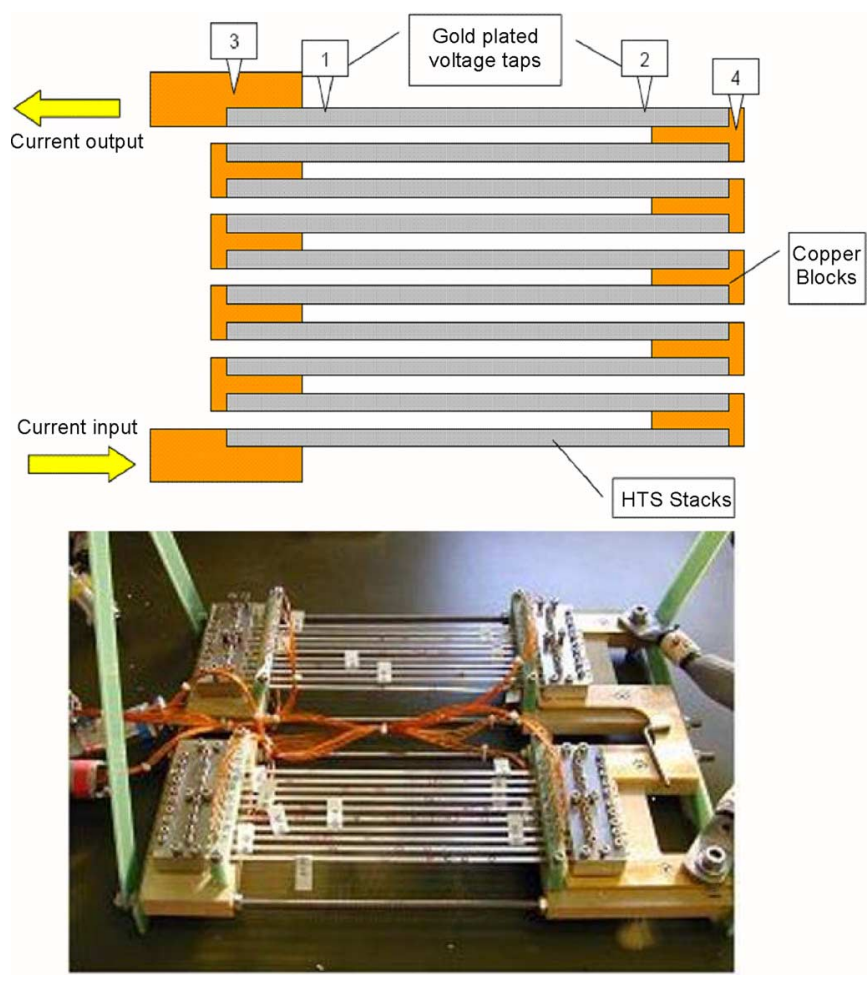

Fig. 2. Sample holder for characterization of HTS stacks. The length of the stacks is $0.35 \mathrm{~m}$.

\section{HTS Stacks Characterization}

The critical current of the HTS stacks was measured in a saturated liquid nitrogen bath and in self-field conditions. Tooling was designed and provided to CESI by CERN, to allow the measurement of up to twenty stacks in series (Fig. 2). Soldering of both electrical connections and voltage taps was avoided in order to minimize the risk of damage and/or contamination of the HTS conductor, already cut to the length required for integration in the leads. The electrical connections between stacks and to the power terminals were made by clamping gold-plated copper blocks to the HTS stacks with a controlled torque of $1.5 \mathrm{Nm}$. Spring loaded needles, pressed against the HTS conductors, were used to measure the voltage drop across a stack length of $0.26 \mathrm{~m}$ for the derivation of the critical current value, while voltage taps built into the copper blocks were used to measure the contact resistance at both ends of each stack. The test procedure was comprised of the following steps:

1) Immersion of the set-up into $\mathrm{LN}_{2}$ with a 15-minute dwell time before starting electrical characterization;

2) Tightening of electrical connections (after cooling, to avoid problems due to differential contraction);

3) Applying current ( $I)$ at steps of $20 \mathrm{~A}$ up to $200 \mathrm{~A}$ or $400 \mathrm{~A}$, depending on the type of stack, and thereafter in 5 A steps up to the maximum current;

4) Unscrewing of electrical connections to stacks (before warming up);

5) Removal of set-up from liquid nitrogen and slow warming-up in an atmosphere of dry nitrogen gas.

The voltage drops used to measure contact resistance and critical current were recorded together with the current value and 


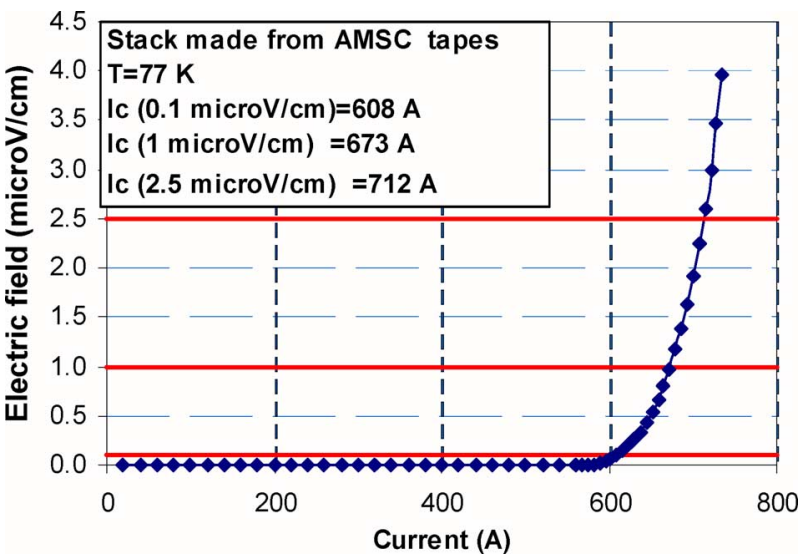

Fig. 3. Example of $E-I$ curve obtained from $I_{c}$ measurements.

the measured temperature of the nitrogen bath. For each stack the critical currents corresponding to electric fields $(E)$ of $0.1,1$, and $2.5 \mu \mathrm{V} / \mathrm{cm}$ were derived (Fig. 3), as well as the $n$-value, calculated as the slope of the logarithmic plot of the voltage versus current in the range from 0.1 to $2.5 \mu \mathrm{V} / \mathrm{cm}$. The resistance of the contact to the stacks was always $\leq 1.5 \mu \Omega$ at $77 \mathrm{~K}$.

\section{MODELING OF HTS STACKS}

The critical current of a stack of tapes is lower than the value obtained by simply summing the critical current of each individual tape. This is due to the increased self-field $(B)$ of the stacked conductor, where it is the field component $\left(B_{\perp}\right)$ perpendicular to the plane of the tape that contributes mainly to the degradation of the electrical performance. The analytical calculation of the self-field of the stack can be done by modeling the superconductor as an infinitely long wire having an elliptic cross-section and carrying a uniform current density. The magnetic field lines are concentric ellipses and the maximum field occurs at the edge of the superconductor. In the case of a stack of 8 tapes, each having a critical current of $120 \mathrm{~A}$, the peak field is $100 \mathrm{mT}$ and decreases to zero at the center of the ellipse. The peak field on a single tape carrying $120 \mathrm{~A}$ is $18 \mathrm{mT}$. From the $I-B_{\perp}$ curves measured at $77 \mathrm{~K}$ on the single tapes, a reduction in critical current of about $60 \%$ might be expected. However, not all the tapes in the stack experience the peak field, and current re-distribution in the stack, due to the magnetic field, plays an important role in the real electrical performance. To take this into account, a finite element analysis has been made on a stack of tapes. Numerical evaluation of the magnetic field and current distribution is required because of the non-linear resistivity $(\rho)$ of the superconducting material, whose dependence on the local current density $(J)$ can be expressed as a power law derived from experimental current-voltage curves of the HTS tapes:

$$
\rho(J)=\left(E_{c} / J_{c}\right) \cdot\left|J / J_{C}\right|^{n-1} .
$$

In (1), $J_{c}$ is the critical current density determined by the electric field criterion $1 \mu \mathrm{V} / \mathrm{cm}$ and the $n$ exponent lies typically in the range 19-25. For the $J_{c}$ dependence of the tapes on the perpendicular field, which is derived from experimental data, (2) was used:

$$
J_{c}(B)=J_{c o} /\left(g-(g-1) \exp \left(-B_{\perp} / B_{0}\right)\right) .
$$
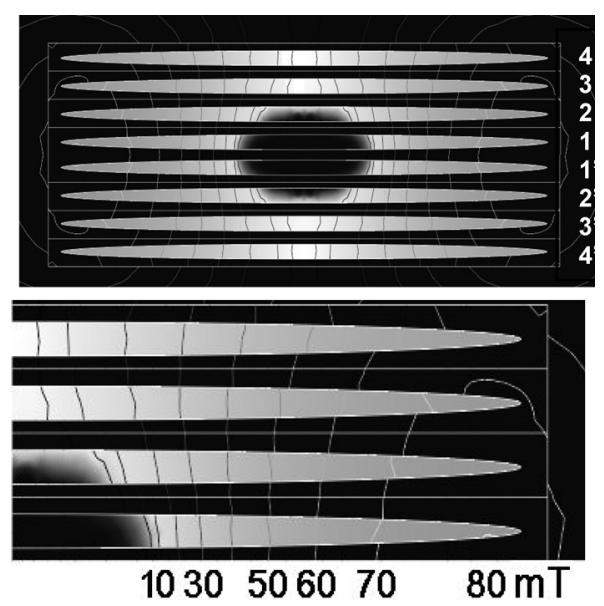

Fig. 4. Results of FEM analysis. $I$ and $B_{\perp}$ contour plot for 8 stacked tapes and $1 / 4$ symmetry. In the simulation it is seen that $B_{\perp}=80 \mathrm{mT}$ at the edge of the middle tape.

TABLE II

CAlculated CurRent Distribution AmONG HTS TAPES With $I_{c}=120 \mathrm{~A}$

\begin{tabular}{ll}
\hline \hline \multicolumn{1}{c}{ HTS Conductor } & \multicolumn{1}{c}{ Current at 77 K (A) } \\
\hline Tapes 1 \& 1' & 57.7 \\
Tapes 2 \& 2' & 77.8 \\
Tapes 3 \& 3' & 89.8 \\
Tapes 4 \& 4' & 91.3 \\
Tapes 1 to 4 & 316.6 \\
Total HTS & 633.2 \\
Matrix contribution & 0.022 \\
Total Stack & 633.22 \\
\hline \hline
\end{tabular}

In (2) $g$ is a fitting parameter (10 in this case) found for individual tapes having $I_{c}=120 \mathrm{~A}$ at $77 \mathrm{~K}$, and $J_{c 0}=3.5 \times$ $10^{8} \mathrm{~A} / \mathrm{m}^{2}$ is the critical current density at $77 \mathrm{~K}$ and self-field.

Fig. 4 shows the current and field distributions calculated for a stack of 8 tapes with critical current of $120 \mathrm{~A}$ at $77 \mathrm{~K}$, in self-field. The geometry of each tape was represented as a superconducting elliptical cross section surrounded by a gold-doped silver matrix. It is evident that the internal tapes $\left(1,1^{\prime}, 2,2^{\prime}\right)$ cannot be fully exploited as the perpendicular field value at the stack edge reaches up to $80 \mathrm{mT}$.

The calculated current distribution among the tapes is summarized in Table II. The internal tapes show the highest reduction of $I_{c}$, but the external tapes $\left(3,3^{\prime}, 4,4^{\prime}\right.$ in Fig. 4) also show a $20 \%$ reduction in critical current. The matrix contribution is negligible. From this analysis it can be seen that taking $8 \times I_{c 0}=960$ A would lead to a $34 \%$ overestimate of capacity of the stack.

\section{EXPERIMENTAL RESULTS AND DISCUSSION}

\section{A. Test Results on Complete Set of Measured Stacks}

The results of the measurements made on 10070 stacks are reported in Fig. 5, where a bin size of 5 A has been chosen for the sampling of the critical current. The three types of stack belong to three critical current populations having mean values of 340 A (EHTS-7), 442 A (EHTS-9) and 627 A (AMSC-8). The parameters of the statistical analysis performed on the full set of measured conductors $(N s t)$ are summarized in Table III, where 


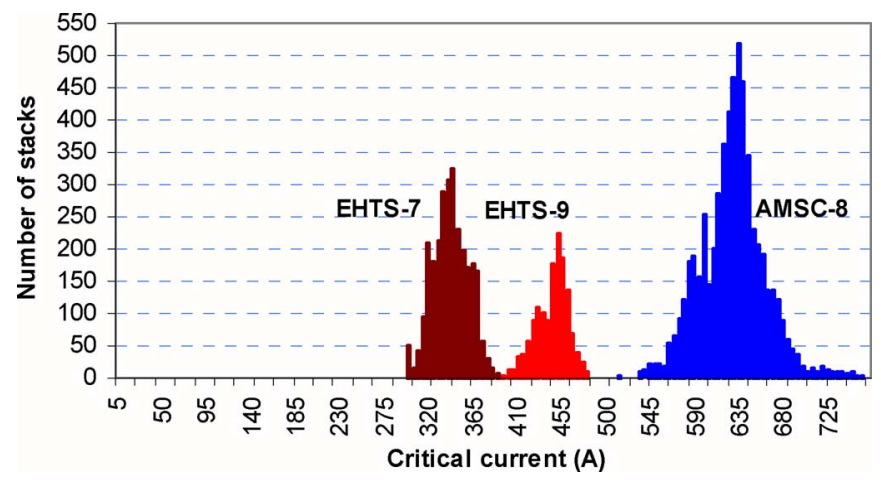

Fig. 5. $I_{c}$ measured at $77 \mathrm{~K}$ and in self-field on $10070 \mathrm{Bi}-2223$ stacks.

TABLE III

Statistical ANalysis Performed on the Complete Series of Stacks

\begin{tabular}{llllll}
\hline \hline & $N s t$ & $\operatorname{Imin}(\mathrm{A})$ & $\operatorname{Imax}(\mathrm{A})$ & $\operatorname{Imean}(\mathrm{A})$ & $C V(\%)$ \\
\hline AMSC-8 & 5870 & 501 & 758 & 627 & 5.1 \\
EHTS-7 & 2780 & 279 & 411 & 340 & 5.5 \\
EHTS-9 & 1420 & 390 & 480 & 441 & 3.6 \\
\hline \hline
\end{tabular}

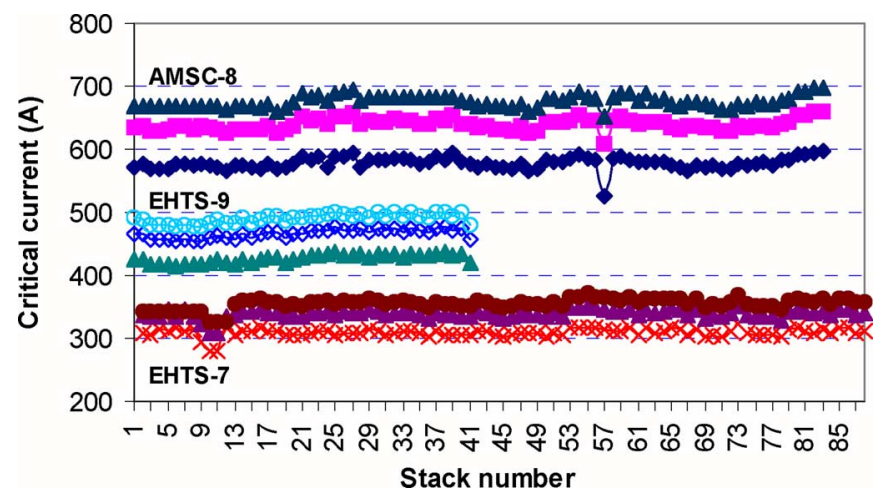

Fig. 6. $I_{c}$ measured on stacks according to $0.1,1$, and $2.5 \mu \mathrm{V} / \mathrm{cm}$ criteria.

the minimum (Imin) and maximum (Imax) values of critical current are given for each stack population, together with the coefficient of variation $(C V)$ and the mean value (Imean). The critical current measurements reported in Fig. 5 were made with the $1 \mu \mathrm{V} / \mathrm{cm}$ electric field criterion. The 0.1 and $2.5 \mu \mathrm{V} / \mathrm{cm}$ electric field criteria gave typical deviations from the $1 \mu \mathrm{V} / \mathrm{cm}$ criterion of about 9 and 5\% respectively. Fig. 6 reports the $I_{c}$ value corresponding to the three electric field criteria as measured on the three types of stack derived from three different spools.

The stacks derived from one specific spool were not useable due to bubbling problems. The porosity of the conductor on this spool was too high, and it was replaced by the manufacturer free of charge. The total number of stacks that did not pass the electrical characterization tests, due to their degraded critical current, was less than $1 \%$ of the total quantity assembled.
TABLE IV

Critical CurRent Measured on TAPES and Derived Stacks

\begin{tabular}{lllll}
\hline \hline & Nst & $\begin{array}{c}\text { Imin } \\
(\mathrm{A})\end{array}$ & $\begin{array}{c}\text { Imax } \\
(\mathrm{A})\end{array}$ & $\begin{array}{c}\text { Imean } \\
(\mathrm{A})\end{array}$ \\
\hline Stacks AMSC-8 from Spool A & 82 & 625 & 660 & 640 \\
Tape AMSC from Spool A & - & 117 & 123 & 120 \\
Stacks EHTS-7 from Spool B & 72 & 324 & 352 & 338 \\
Tape EHTS from Spool B & - & 73 & 79 & 76 \\
Stacks EHTS-9 from Spool C & 41 & 453 & 477 & 466 \\
Tape EHTS from Spool C & - & 81 & 88 & 85 \\
\hline \hline
\end{tabular}

\section{B. Tape and Stacks Critical Current}

Each HTS stack was made using tape from the same spool. The homogeneity in critical current of the stacks derived from the same spool reflects the homogeneity in the critical current of the spool, for which a maximum variation of $\pm 5 \%$ from the mean value has been measured. The reduction in critical current of the AMSC stacks with respect to the value obtained from the number of tapes multiplied by their individual critical current has been measured to be in the range of 32-35\%; for the EHTS stacks, the reduction was in the range 34-39\%. These figures are largely in agreement with the calculations discussed in Section III.

In Table IV the critical current measured on the three types of stack derived from three different spools are compared to the critical currents of the tape, indicating the level of spread.

The average $n$ value of the EHTS-7 stacks, derived from the measurements in the range from 0.1 to $2.5 \mu \mathrm{V} / \mathrm{cm}$, was 22 . Due to the increased self-field of the conductors, it is lower than for the tape, for which the average value was 30 .

\section{CONCLUSION}

The series of more than $10000 \mathrm{Bi}-2223$ stacks needed for the LHC current leads has been assembled at CERN. Their electrical characterization, made at CESI, has confirmed the good electrical quality of the conductors. The data obtained from these measurements, performed on a relatively large scale production, are excellent as far as the homogeneity of the electrical properties of both tape and stacks is concerned.

\section{ACKNOWLEDGMENT}

The authors wish to thank Lionel Poyet and Valerio Rossi for their professional support in the realization of the required tooling and in the performance of the electrical measurements.

\section{REFERENCES}

[1] A. Ballarino, S. Mathot, and D. Milani, " 13000 A HTS current leads for the LHC accelerator: From conceptual design to prototype validation," in Proc. Eucas 2003, IOP, 2003.

[2] A. Ballarino, "HTS in the LHC \& in the LHC upgrades," in Proceedings of WAMDO Workshop, CERN, April 2006 [Online]. Available: http://wamdo-2006.web.cern.ch/wamdo-2006

[3] F. Grilli, L. Martini, S. Stavrev, B. Dutoit, and R. Brambilla, "Analysis of magnetic field and geometry effects for the design of HTS devices for AC power applications," IEEE Trans. Appl. Supercond., vol. 15, no. 2, pp. 2074-2077, 2005. 